\title{
Exercise Treatment Effect: The Brain Metabolite Change of Magnetic Resonance Spectroscopy in Intracerebral Haemorrhage Rat Model
}

\author{
Suk-Jun Lee ${ }^{1}$, Seung-Man Yu ${ }^{2}$ \\ ${ }^{1}$ Department of Biomedical Laboratory Science, College of Health Science, \\ Cheongju University of Korea \\ ${ }^{2}$ Department of Radiological Science, Gimcheon University of Korea \\ Gyeongsangbuk-do 39528, Gimcheon, Republic of Korea \\ ysm9993@gmail.com
}

\section{Extended Abstract}

Intracerebral haemorrhage ( $\mathrm{ICH})$ consisting in an acute and spontaneous extravasation of blood into the brain parenchyma accounts for 10-15\% of all stroke cases and results in the highest rates of mortality and disability of all stroke subtypes [1]. Magnetic resonance spectroscopy (MRS) has been widely used to diagnose neuro-disease. MRS is a noninvasive and in vivo method with high sensitivity and specificity [2]. The objective of this study were to examine the brain metabolite concentration quantification change by in-vivo ${ }^{1} \mathrm{H}-\mathrm{MRS}$ analysis in animal haemorrhage model, and we determined the bio-marker that was shown the effect of exercise treatment in haemorrhage disease.

The 6-weeks-old 24 Sprague-Dawley rats were anaesthetized with chloral hydrate $(400 \mathrm{mg} / \mathrm{kg}$, i.p.) and placed in a stereotactic frame, collagenase injected over the next $5 \mathrm{~min}$ using a pump. The experimental group 12rats were conducted treadmill training. The training program of this research was implemented in 2 weeks with 55 to 85 percent of $\mathrm{VO}_{2} \mathrm{max}$ and during determined period $15 \mathrm{~min}$ one a day. All MRI and 1H-MRS experiments were performed on a 3.0Tesla MRI scanner (Achiva Tx 3.0 T; Philips Medical Systems, Netherlands) with a maximum gradient of $200 \mathrm{mT} / \mathrm{m}$ using a 4-channel animal coil (CG-MUC18-H300-AP, Shanghai Chenguang Medical Technologies Co., Ltd., China). A $8 \times 6 \times 6 \mathrm{~mm}^{3}$ voxel was placed within a whole brain parenchyma with hemorrhage area. The ${ }^{1} \mathrm{H}-\mathrm{MRS}$ raw data were analyzed using LCModel software (version 6.3- ${ }^{1} \mathrm{H}$, Stephen W. Provencher). The integrating areas under peaks were measured as follows: glutamate (Glu), glutamine (Gln), Choline-containing Compounds (tCho, phosphoryl choline + glycerophosphochline), N-Acetyl Aspartate (NAA), N-Acetyl Aspartyl Glutamate (NAAG). Less than 15\% standard deviation (\%SD) of metabolite quantification data was allowed. The $\% \mathrm{SD}$ called the Cramér-Rao lower bound of useful reliability indicators was used for error estimates. The concentration value of ( $\mathrm{Glu}+\mathrm{Gln}) / \mathrm{tCr}$ at experimental group after applying the exercise treatment was $1.838 \pm 0.606$, which was significantly $(\mathrm{p}=0.021)$ higher than control group $(1.270 \pm 0.283)$. Although the Glu concentration of experimental group $(1.340 \pm 0.197)$ was higher than control group $(1.153 \pm 0.132)$, but there was statically insignificant $(\mathrm{p}=0.067)$. The $\mathrm{tCho} / \mathrm{tCr}$ concentration value of experimental group was $0.267 \pm 0.012$, which was significantly $(\mathrm{p}=0.000)$ lower than control group $(0.352 \pm 0.051)$. The NAA/tCr and NAAG/tCr of concentration could not separate, the (NAA+NAAG)/tCr concentration value for experimental and control group were $1.258 \pm 0.226$ and $1.2742 \pm 0.093$ respectively. No significant difference in the concentration levels of experimental and control group were observed $(\mathrm{p}=0.839)$.

There was great significance in revealing that $(\mathrm{Glu}+\mathrm{Gln}) / \mathrm{tCr}$ value was increased, and $\mathrm{tCho/tCr}$ concentration level was decrease applying exercise treatment methods on hemorrhage animal model. Therefore, the metabolite concentration change of $(\mathrm{Glu}+\mathrm{Gln}) / \mathrm{tCr}$ and $\mathrm{tCho} / \mathrm{tCr}$ can be used as a powerful bio-marker that represented an exercise treatment in hemorrhage patients.

\section{References}

[1] V. Beray-Berthat, C. Delifer, V. C. Besson, H. Girgis, B. Coqueran, M. Plotkine, C. Marchand-Leroux, and I. Margaill, "Long-term histilogical and bevavioural characterization of a collagenase-induced model of intracerebral haemorrhage in rats," J. Neuro Methods., vol. 191, pp. 180-190, 2010.

[2] V. Govindaraju, K. Young, and A. Maudsley. "Proton NMR chemical shifts and coupling constants for brain metabolites," NMR in Biomed., vol. 13, pp. 129-153, 2000. 